\title{
Cecilia Anichini
}

\author{
Siena Academy of Sciences, University of Siena
}

\begin{abstract}
Prostate cancer (PCa) is the second most common cancer in men worldwide. However the etiology of this disease remains largely unclear. Both genetic and environmental factors seem to be involved in the pathogenesis. In the last years the role of reactive oxygen species (ROS) in deterioration of DNA, proteins, lipids of membrane cells has been demonstrated. Also external antioxidant factors such as vitamin $\mathrm{C}$ and vitamin $\mathrm{E}$, melatonin, that intercept free radicals, play a role, preventing damage to cellular biomolecules. The antioxidant enzyme glutathione peroxidase 1 (GPX1) is a part of the enzymatic antioxidant defence, preventing oxidative damage to DNA, proteins and lipids by detoxifying hydrogen and lipid peroxides that may contribute to prostate cancer development. Some recent studies indicate an association between GPX1 Pro198Leu polymorphism and an increased risk of cancer. So the alimentary integration with an antioxidant agent such as ascorbate of potassium with ribose (PAR) can play an important rule in the prevention and also cure of prostatic cancer, opposing the effect of ROS, both in men carriers of the polymorphism the GPX1 Pro198Leu and in the general population after the demonstration on blood samples of an increase of the oxidative stress parameters.
\end{abstract}

Keywords: cancer, prostate, genes, oxidative stress

Prostate cancer is the second most common cancer in men worldwide. However the cause of this disease remains largely unclear. Both genetic and environmental factors seem to be involved in the pathogenesis.

Polymorphic genes have been demonstrated to be involved in the pathogenesis: in particular Genome-wide and replication association studies (GWAs) have identified multiple loci at which common variants modestly influence the risk of developing prostate cancer Significant associations were found between 31 SNPs and PCa. $(1,2)$

In the last years the role of reactive oxygen species (ROS) in deterioration of DNA, proteins, lipids of membrane cells has been demonstrated. In fact the generation of reactive oxygen species may be both beneficial to cells, performing a function in inter- and intracellular signalling, and detrimental, modifying cellular biomolecules, accumulation of which has been associated with numerous diseases. $(3,4,5)$

Oxidative stress is a process that occurs when cells tissues and biomolecules undergo an excessive exposition to free radical species causing a number of human pathologies, such as carcinogenesis, cardiovascular and neurodegenerative diseases (6). There is evidence that suggests that oxidative stress plays a role also in prostatic carcinogenesis. The living cells are constantly exposed to potentially damaging free radical species, whose origin may be both intracellular arising from cellular metabolism and extracellular, produced by exposure to chemical, physical and infective factors (ion- izing and ultraviolet radiations, drugs, viruses and bacterial diseases, smoke, air pollution, emotional stress). The reactive oxygen species (ROS) include superoxide radical $(\mathrm{O})$, reactive hydroxil-radical $(\mathrm{OH})$, non radical hydrogen peroxide ( $\mathrm{H} 2 \mathrm{O} 2)$. ROS have as cellular target DNA, protein and lipids of cellular membrane (7). To combat attack from ROS, and other free radicals, living cells have acquired a number of defences: enzymes, such as superoxide desmutase, catalase and glutathione peroxidase limit the levels of ROS. Also external antioxidant factors such as such as vitamin $C$, and vitamin $\mathrm{E}$, melatonin, that intercept free radicals, play a role, preventing damage to cellular biomolecules. The antioxidant enzyme glut, preventing oxidative damage to DNA, proteins and lipids by detoxifying hydrogen and lipid peroxides that may contribute to prostate cancer development. A number of human cancers demonstrate low levels of intracellular antioxidant enzymes (catalase, glutathione peroxidase) and antioxidant molecules (vitamin $E$, vitamin $C$, and vitamin A, melatonin, curcumin). Some recent studies indicate an association between GPX1 Pro198Leu polymorphism and an increased risk of cancer and older ages may influence the association between GPX1 Pro198Leu polymorphism and PCa (8).

Such cancer cells are unable to detoxify large quantities of $\mathrm{H} 2 \mathrm{O} 2$. Thought generalizations about antioxidants cannot be made. Research has demonstrated the anticancer activity of vitamin $\mathrm{C}$ (VC) for some decades. The $\mathrm{VC}$ has been performed positively in vitro for prostate 
cancer, breast cancer, ovarian cancer, bladder cancer, hepatocarcinoma, leukemias. Nevertheless human trials with oral VC show modest benefit, also if more than the other anticancer antioxidants. (Anichini $C$ et all Anticancer research 2011). While there are many reports on the anticancer effects of various natural agents, there is little information available on results of combining these agents. Some agents that are classified as antioxidants have been a evaluated to verify the effect in comparison or association to the standard chemotherapies. Indeed the alimentary integration with a combined antioxidant agent such as Ascorbate of Potassium with Ribose (PAR) plays an important rule in the prevention and also cure of prostatic cancer, opposing the effect of ROS, both in men carriers of the polymorphism the GPX1 Pro198Leu and in the general population after the evidence, on blood samples, of an increase of the oxidative stress parameters $(9,10,11)$. While in the past time an empiric use of those integrators was used, now a more correct administration is possible, after the determination of some reliable OS biomarker profile in a blood sample in order to compare its values with the ones of control(14). Biomarker profile included advanced oxidation protein products (AOPP), non protein binding iron (NPBI), isoprostanes (IP2), carbonyl groups (CO) and hydroperoxides (dRO). The biomarker profile and PAR administration have been duly approved by local Ethics Committee (C. Anichini Research Atheneum Progect 2005).

A protective action of ascorbate of potassium with ribose has just been confirmed on cellular lines of healthy breast fibroblasts(HTB 125) exposed to radiation with 200 RadLe Healthy radiated cells not treated with PAR showed a slower growth vetocity and duplication with morphological aspect changes, while cells radiated and in a medium with PAR had normal growth, duplication and aspects. $(15,16)$.

A therapy with the antioxidant ascorbate of potassium with ribose (Ac.L-Ascorbic mg150, D-Ribose mg3) may reduce cancer development and helps during chemotherapy and Rx therapy, that induces a greater production of free radicals (12).

Prevention and therapy of prostate cancer with PAR may be useful in:

1- patients with the polimorphism GPXI;

2- prostate adenoma;

3- prostate cancer;

4- familiarity for prostatic cancer;

5- tumour in other organs with the risk to develop

a second cancer.

\section{REFERENCES}

1. Liu H, Wang B, Han C. Meta-analysis of genome-wide and replication association studies on prostate cancer. Prostate. 2011 Feb 1;71(2):209-24. doi: 10.1002/pros.21235.

2. Zheng SL, Sun J, Wiklund F, Smith S, Stattin P, Li G, Adami HO, Hsu FC, Zhu Y,囚Bälter K, Kader AK, Turner AR, Liu W, Bleecker ER, Meyers DA, Duggan D, Carpten $\bowtie J D, C h a n g ~ B L$, Isaacs WB, Xu J, Grönberg H.囚Cumulative association of five genetic variants with prostate cancer. $\triangle N$ Engl J Med. 2008 Feb 28;358(9):910-9. Epub 2008 Jan 16.

3. Halliwell B. Reactive oxygen species in living system: source, biochemistry and role in human disease. Am J Med. 1991;91(suppl.3C):14-22.

4. Del Maestro R. An approach to free radicals in medicine and biology. Acta Physiol Scand. 1992;492:153-68.

5. Halliwell B. Free radicals, antioxidants and human disease: curiosity, cause or consequence? Lancet. 1994;344:721-4

6. Farooqi T, Farooqi AA. Lipid-mediated oxidative stress and inflammation in the pathogenesis of Parkinson's disease. Parkinsons Dis. 2011 Feb 15;2011:247467.

7. Erdem O, Eken A, Akay C, Arsova-Sarafinovska Z, Matevska N, Suturkova L, Erten K, Ozgök Y, Dimovski A, Sayal A, Aydin A. :Association of GPX1 polymorphism, GPX activity and prostate cancer risk, Hum Exp Toxicol, 2011 Jun 2.

8. Jacob RA. The integrated antioxidant system. Nutr Res.1995;15:75566.

9. Valsè Pantellini G. Breve cenno sulla genesi dei tumori e sopra una eventuale terapia dei medesimi con Sali di potassio e in particolare con ascorbato di potassio. Rivista di Patologia Clinica. 1970 XXV (5):219225.

10. Lamson Davis W. The Vitamin C:Vitamin K3 System - Enhancers and Inhibitors of the Anticancer Effect, , Alternative Medicine Review Volume 15, Number 4, 2010

11. Vissers MC, Gunningham SP, Morrison MJ, Dachs GU, Currie MJ.: Modulation of hypoxia-inducible factor-1 alpha in cultured primary cells by Intracellular ascorbate, Free Radical Biology $\theta$ Medicine (2007)

12. Bera et all. Dexametasone induced oxidative stress enhances Myeloma cell radiosensitization -Neoplasia (2010)

13. Bruni et all Bologna sett 2010 Congresso Naz. Soc. Italiana Fisica 14. Anichini C, Lo Rizzo C, Longini, M, Paoli G, Rosanna Maria Di Bartolo R M, Proietti F, Buonocore G. Beckwith-Wiedemann Syndrome: Potassium Ascorbate with Ribose Therapy in a Syndrome with High Neoplastic Risk. Anticancer Research. [in press]

15. Paoli $G$. The biomagnetic nature of cancer and the role of Potassium Ascorbate and Ribose against cellular degeneration, Journal of New Energy - An international Journal of New Energy Systems Vol 7, $n^{\circ}$ 3, 2003

16. Croci S, Bruni L, Bussolati S, Castaldo $M$ and Dondi: Potassium bicarbonate and D-ribose effects on A72 canine and HTB-126 human cancer cell line proliferation in vitro"; M Cancer Cell International" 2011, 11:30, Published on 22 August 2011 : 